\title{
A standard grid for density functional calculations
}

\author{
Peter M.W. Gill ', Benny G. Johnson and John A. Pople \\ Department of Chemistry, Carnegie Mellon University, Pittsburgh, PA 15213, USA
}

Received 20 April 1993; in final form 12 May 1993

\begin{abstract}
An efficient and reasonably accurate grid, designated SG-1, is proposed for use in density functional calculations. Defined for all atoms from $\mathbf{H}$ to Ar, SG-1 is recommended as a standard grid, analogous to the various standard basis sets which are used in contemporary quantum chemistry. In calculations on systems of moderate size, the differences between SG-1 and very large grids are of the order of $0.2 \mathrm{kcal} / \mathrm{mol}$, yet SG-1 is sufficiently small to be applied to large systems.
\end{abstract}

\section{Introduction}

In recent years, the Kohn-Sham (KS) version of density functional theory [1] has gained widespread acceptance [2-4] as a remarkably cost-effective tool for computational quantum chemistry. It is distinguished from conventional ab initio methods by its treatment of the exchange and correlation contributions to the electronic energy, which are expressed (approximately) as three-dimensional integrals (over all space) of analytic functions [5-10] of the alpha and beta densities $\left(\rho_{\alpha}, \rho_{\beta}\right)$ and, sometimes, of their derivatives. In this way, the exchange-correlation energy

$E_{\mathrm{XC}}=\int F\left(\rho_{\alpha}(\boldsymbol{r}), \rho_{\beta}(\boldsymbol{r}), \nabla \rho_{\alpha}(\boldsymbol{r}), \nabla \rho_{\beta}(\boldsymbol{r}), \ldots\right) \mathrm{d} \boldsymbol{r}$

becomes essentially a one-electron integral, notwithstanding the fact that it arises from many-electron interactions. There is a price to be paid, however, for this extremely convenient simplification - the function $F$ is invariably too awkward to permit (1) to be evaluated in closed form - and, consequently, one must resort to three-dimensional numerical integration techniques.

Many KS computer programs [11-16] begin by

1 Present address: Department of Chemistry, Massey University, Palmerston North, New Zealand. E-mail address: gill@rush.chem.cmu.edu. partitioning the multi-center molecular integral (1) into single-center atomic subintegrals and then compute each of the latter numerically on an atomic grid. We have adopted the partitioning scheme developed by Becke in section 3 of ref. [17] but we have not adopted the "atomic size adjustments" which he recommends in his Appendix. Normally, the atomic grids consist of points on concentric spheres about each atom but little effort appears to have been made to standardize, or even to publish in detail, the exact atomic grids that various groups use. If the grids are sufficiently dense, the computed energy will converge towards the result for complete integration of the expression (1). However, for finite grids, it is desirable to have a well-defined specification of the points and weights, so that a precise energy is obtained from a given functional, a given orbital expansion basis (if used) and a given grid. The principal purpose of this Letter is to propose and test a standard grid (designated SG-1), which has been incorporated into the Q-Chem program [16] and into a modified version of the GAUSSIAN 92 program [18]. In conjunction with an exchange-correlation functional and basis set, such a grid completes the specification of a Kohn-Sham theoretical model chemistry [19].

Before getting into detail, we should note some inherent limitations of grid-based models. The angular portions of any atom-based grid have to be oriented relative to Cartesian axes and, therefore, oriented in some manner relative to the orientation of the mol- 
ecule. It follows that specification of a standard grid requires specification of a standard orientation of the nuclear coordinates. Such a definition, which ensures that the energy does not depend on the userspecified orientation, will be presented in the following sections. However, the possibility that the axes may switch in some discontinuous manner cannot be excluded, so the resulting potential surface may possess some discontinuities. This situation is particularly probable in molecules with nuclear structures at, or close to, high-symmetry configurations. Such discontinuities become smaller as the grid becomes larger, but they cannot easily be climinated.

A second important general point concerns molecules with electrons assigned to symmetry-degenerate molecular orbitals, such as the e orbitals in $\mathrm{C}_{3 \mathrm{v}}$ ammonia. If the integration in ( 1 ) is carried out exactly, the resulting energy is invariant to unitary transformations within such sets of orbitals. However, once a finite grid is used, invariance is lost; to achieve a unique result, it is necessary to fix the individual orbitals in some well-defined manner. Thus, for molecules with non-abelian symmetry, we need to specify the electronic, as well as the nuclear, orientation.

For both nuclei and electrons, therefore, some arbitrary but well-defined choices of orientation have to be imposed. These are specified in Appendices $A$ and $B$.

\section{Euler-Maclaurin-Lebeder grids}

Suppose that we wish to devise a quadrature formula to approximate

$$
\begin{aligned}
I & =\int F(\boldsymbol{r}) \mathrm{d} \boldsymbol{r} \\
& =\int_{0}^{\infty} \int_{0}^{\pi} \int_{0}^{2 \pi} r^{2} \sin \vartheta F(r, \vartheta, \phi) \mathrm{d} \phi \mathrm{d} \vartheta \mathrm{d} r .
\end{aligned}
$$

Traditionally, and for simplicity, the radial and angular integrations are separated by employing product quadrature formulae, i.e.

$I \approx \sum_{i=1}^{N^{r}} w_{i}^{r} \sum_{j=1}^{N^{\Omega}} w_{j}^{\Omega} F\left(r_{i}, \vartheta_{j}, \phi_{j}\right)$, in which $N^{r}$ and $N^{\Omega}$ are the numbers of radial and angular points, respectively, and the $w_{i}^{r}$ and $w_{j}^{\Omega}$ are radial and angular weights, respectively. Although it is inefficient [20] to continue in this way to separate the two angular integrations, i.e.

$I \approx \sum_{i=1}^{N r} w_{i}^{r} \sum_{j=1}^{N^{\theta}} w_{j}^{\theta} \sum_{k=1}^{N \phi} w_{k}^{\phi} F\left(r_{i}, \vartheta_{j}, \phi_{k}\right)$

the even greater simplicity of (4) has led Murray, Handy and Laming (MHL) to recommend it [21]. We recommend (3) for routine work but will use (4) to obtain "benchmarks" for very large grids.

The inner sum in (3) corresponds to quadrature on the surface of a sphere and the most sophisticated results in this area are those obtained by Lebedev [22] and Konyaev [23] whose grids have octahedral and icosahedral symmetry, respectively. We prefer the Lebedev grids because they are better suited to the treatment of Cartesian basis functions in molecular systems with abelian symmetry. A grid of degree / exactly integrates all spherical harmonics of degree $l$ or less and, thus, is a two-dimensional analogue of the more familiar Gauss-Legendre grid in one dimension [24]. Lebedev has published grids up to $l=29$ and we will be particularly interested in the $l=3,9,15,23$ and 29 grids which have $N^{\Omega}=6$, $38,86,194$ and 302 points, respectively.

Given $\left(w_{j}^{\Omega}, \vartheta_{j}, \phi_{j}\right)$ values from Lebedev, we now address the problem of selecting $w_{i}^{r}$ and $r_{i}$ values, i.e. we seek a quadrature formula of the form

$$
\int_{0}^{\infty} r^{2} G(r) \mathrm{d} r \approx \sum_{i=1}^{N r} w_{i}^{r} G\left(r_{i}\right),
$$

where, for convenience, we will assume that $G$ is bounded at $r=0$ and decays at least exponentially for large $r$. A number of solutions to this have been proposed by previous authors [12-14,17,25] but, in preference to these, we have adopted the EulerMaclaurin scheme [21] recently introduced by Murray, Handy and Laming but based on much earlier work [26] by Handy and Boys. In this scheme, one uses

$$
\begin{aligned}
& w_{i}^{r}=2 R^{3}\left(N^{r}+1\right) i^{5}\left(N^{r}+1-i\right)^{-7}, \\
& r_{i}=R i^{2}\left(N^{r}+1-i\right)^{-2},
\end{aligned}
$$

where $R$, which we term the "atomic radius", is a 
measure of the radial extent of the atom in question.

We will refer to an atomic grid which uses an $N^{r_{-}}$ point Euler-Maclaurin grid in conjunction with an $N^{\Omega}$-point Lebedev grid as EML- $\left(N^{r}, N^{\Omega}\right)$ or simply $\left(N^{r}, N^{\Omega}\right)$. For example, in some of our earlier papers [27-29] we have employed $(50,194)$, and have found it uniformly satisfactory for calculation of molecular energies and properties. We will refer to an atomic grid which uses an $N^{r}$-point Euler-Maclaurin radial grid in conjunction with an $N^{\theta}$-point Legendre theta grid and an $N^{\phi}$-point even-spaced phi grid [21] as MHL- $\left(N^{r}, N^{\theta}, N^{\phi}\right)$ or simply $\left(N^{r}, N^{\theta}\right.$, $N^{\phi}$ ). We have chosen to base our standard grid on an EML and, therefore, our first task is to determine which EML yields acceptably small grid errors for some energies of chemical interest.

Of course, we must first define what we mean by an "acceptably small" grid error. We will regard a grid error as acceptable if it is $\approx 0.2 \mathrm{kcal} / \mathrm{mol}$ or $\approx 300 \mu$ hartree for a medium-sized molecule since this is significantly smaller than the accuracy typically achieved by contemporary quantum chemical procedures. We note, however, that any grid's error will inevitably rise as one treats larger and larger molecules.

Before proceeding further, it is necessary to specify the atomic radii $R$ which we use. We described these briefly in an earlier paper [30] but we have not previously listed them explicitly. We define the atomic radius of an atom to correspond to the maximum of the radial probability function $4 \pi r^{2} \varphi^{2}(r)$ of the valence atomic orbital $\varphi(r)$ given by Slater's wellknown rules [31]. The atomic radii which follow from this definition are given in table 1 for the atoms $\mathrm{H}$ to $\mathrm{Ar}$.

Of a wide variety of chemical problems which we have examined, we have found that the (trans)- $n$ butane to iso-butane and (trans, trans)- $n$-pentane to neo-pentane isomerization energies are particularly sensitive tests of the deficiencies of a grid and we shall confine our attention, in the first instance, to these rather demanding test cases. Their difficulty stems from the fact that, although the pairs of isomers are different in shape ( $n$-pentane, for example, is more or less linear while neo-pentane is globular), the density-functional isomerization energies are only of the order of $1 \mathrm{kcal} / \mathrm{mol}$ and can easily be swamped by grid error. (We note that the experimental isomerization energies are roughly 4 and $2 \mathrm{kcal} / \mathrm{mol}$, respectively. However although our DFT isomerization energies are clearly not in good agreement with experiment, this is not relevant to the concerns of this Letter.) We have used a modified version of GAUSSIAN 92 to compute the isomerization energies using the fully self-consistent B-LYP/6-31G* procedure $[27-30,32]$ at $\mathrm{HF} / 6-31 \mathrm{G}^{*}$ geometries and we list them, for various grids, in tables 2 and 3 .

In a recent study [33] of benzene using our B-LYP procedure, Handy et al. showed that a MHL- (96, 32, 64) grid yielded a grid error of $<10^{-7}$ hartree for this molecule. On this basis, we will use this grid as a "benchmark" against which to compare smaller grids. In this way, we find from tables 2 and 3 that $(40,194)$ and $(50,194)$ are the smallest grids yielding an acceptable error for the butane and pentane isomerizations, respectively, while, at the other end of the spectrum, we find that $(30,86)$ gives isomerization energies which are too large by more than a factor of two. Wc also note that, whereas the radial quadrature converges fairly smoothly, convergence of the angular quadrature is much more jagged: such behavior is commonly observed.

Table 1

Atomic radii (bohr) used in the SG-1 grid

\begin{tabular}{lllllll}
\hline atom & $\mathrm{H}$ & $\mathrm{He}$ & $\mathrm{Li}$ & $\mathrm{Be}$ & $\mathrm{B}$ & $\mathrm{C}$ \\
radius & 1.0000 & 0.5882 & 3.0769 & 2.0513 & 1.5385 & 1.2308 \\
atom & $\mathrm{N}$ & $\mathrm{O}$ & $\mathrm{F}$ & $\mathrm{Ne}$ & $\mathrm{Na}$ & $\mathrm{Mg}$ \\
radius & 1.0256 & 0.8791 & 0.7692 & 0.6838 & 4.0909 & 3.1579 \\
atom & $\mathrm{Al}$ & $\mathrm{Si}$ & $\mathrm{P}$ & $\mathrm{S}$ & $\mathrm{Cl}$ & $\mathrm{Ar}$ \\
radius & 2.5714 & 2.1687 & 1.8750 & 1.6514 & 1.4754 & 1.3333 \\
\hline
\end{tabular}


Table 2

Isomerization energy a of iso-butane $\rightarrow n$-butane using various grids

\begin{tabular}{ccccccc}
\hline$N^{r}$ & $N^{\Omega}=86$ & $N^{\infty}=110$ & $N^{\Omega}=146$ & $N^{\infty}=194$ & $N^{\infty}=302$ & $N^{\infty}=32, N^{\infty}=64$ \\
\hline 30 & 1595 & 1405 & 1629 & 1130 & 1052 \\
40 & 1011 & 938 & 1168 & 742 & 706 \\
50 & 903 & 856 & 1117 & 692 & 664 \\
60 & 878 & 839 & 1109 & 686 & 661 \\
70 & 877 & 838 & 1106 & 687 & 662 & 660 \\
96 & & & & & \\
\hline
\end{tabular}

a) In whartree at the B-LYP/6-31G*//HF/6-31G* level of theory.

Table 3

Isomerization energy ${ }^{\text {a }}$ of neo-pentane $\rightarrow n$-pentane using various grids

\begin{tabular}{|c|c|c|c|c|c|c|}
\hline$N^{r}$ & $N^{R}=86$ & $N^{0}=110$ & $N^{2}=146$ & $N^{a}=194$ & $N^{x}=302$ & $N^{\theta}=32, N^{\phi}=64$ \\
\hline 30 & 1925 & 1637 & 2931 & 2386 & 2258 & \\
\hline 40 & 2153 & 1117 & 1798 & 1231 & 1062 & \\
\hline 50 & 2041 & 1015 & 1546 & 1040 & 870 & \\
\hline 60 & 2053 & 926 & 1475 & 1020 & 858 & \\
\hline 70 & 2081 & 947 & 1501 & 1038 & 859 & \\
\hline 96 & & & & & & 758 \\
\hline
\end{tabular}

a) In $\mu$ hartree at the B-LYP $/ 6-31 \mathrm{G}^{*} / / \mathrm{HF} / 6-31 \mathrm{G}^{*}$ level of theory.

\section{The standard SG-1 grid}

Having seen that EML- $(50,194)$ can yield quantitatively reliable results even for a difficult problem such as the pentane isomerization, it would not be unreasonable to propose this as the standard grid and proceed to test it. However, EML- $(50,194)$ is rather large (roughly an order of magnitude larger, for example, than the default grid in the DGauss program [14]) and it would obviously be desirable, if possible, to reduce its size while maintaining its effectiveness. The critical modification of (3) which we will introduce is to allow $N^{\Omega}$ to become dependent on $i$, that is, to usc diffcrent Lebedev grids on different concentric spheres. This strategy, which we term "grid pruning", has previously been considered [21], and is motivated by the realization that, as one approaches a nucleus from the valence region in a molecule, the electron density becomes progressively more spherically symmetrical and can therefore be treated satisfactorily by progressively less sophisticated angular grids.

Given four numbers $\left\{\alpha_{1}, \alpha_{2}, \alpha_{3}, \alpha_{4}\right\}$ and an atomic radius $R$, the four spheres of radius $\alpha_{1} R, \alpha_{2} R, \alpha_{3} R$ and $\alpha_{4} R$ obviously partition an atom into five regions. Within each of these regions, from innermost to outermost, we employ Lebedev grids with 6,38 , 86,194 and 86 points, respectively. Since we have already defined $R$, it only remains to choose $\alpha$ s for each atom. We have selected $\alpha$ values by pruning as aggressively as possible subject to the constraint that the $(50,194)$ and SG-1 energies for the normal-valent hydrides of the atoms from $\mathrm{H}$ to Ar differ by only a few $\mu$ hartree. The resulting values, which are given in table 4, complete the specification of the SG-1 grid for the atoms $\mathrm{H}$ to $\mathrm{Ar}$.

Table 5 lists the B-LYP/6-31G* total energies afforded by various grids. From inspection of this table, it appears that SG-1 yields the same (within a few $\mu$ hartree) energies as EML- $(50,194)$ for a wide

Table 4

Partitioning parameters used in the SG-1 grid

\begin{tabular}{lllll}
\hline Atom & $\alpha_{1}$ & $\alpha_{2}$ & $\alpha_{3}$ & $\alpha_{4}$ \\
\hline $\mathrm{H}-\mathrm{He}$ & 0.2500 & 0.5000 & 1.0000 & 4.5000 \\
$\mathrm{Li}-\mathrm{Ne}$ & 0.1667 & 0.5000 & 0.9000 & 3.5000 \\
$\mathrm{Na}-\mathrm{Ar}$ & 0.1000 & 0.4000 & 0.8000 & 2.5000 \\
\hline
\end{tabular}


Table 5

Total energies ${ }^{a, b)}$ of various molecules ${ }^{\text {) }}$ using various grids d)

\begin{tabular}{|c|c|c|c|c|c|}
\hline Molecule & & $(96,32,64)$ & $(30,86)$ & $(50,194)$ & SG-1 \\
\hline $\mathrm{H}_{2}$ & & -1.165182 & 18 & 0 & -2 \\
\hline $\mathrm{He}$ & & -2.897845 & 44 & 1 & 1 \\
\hline $\mathrm{LiH}$ & & -8.066115 & -48 & 1 & 0 \\
\hline $\mathrm{BeH}_{2}$ & & -15.891924 & -126 & -5 & 3 \\
\hline $\mathrm{BH}_{3}$ & & -26.578759 & 154 & 0 & 0 \\
\hline $\mathrm{CH}_{4}$ & & -40.478861 & -154 & -53 & -52 \\
\hline $\mathrm{NH}_{3}$ & & -56.518234 & 60 & 9 & 9 \\
\hline $\mathrm{H}_{2} \mathrm{O}$ & & -76.388318 & 119 & 11 & 9 \\
\hline $\mathrm{HF}$ & & -100.404440 & -496 & 2 & 3 \\
\hline $\mathrm{Ne}$ & & -128.879568 & -361 & 0 & 0 \\
\hline $\mathrm{NaH}$ & & -162.834957 & 5800 & -26 & -28 \\
\hline $\mathrm{MgH}_{2}$ & & -201.221105 & -1296 & -9 & -22 \\
\hline $\mathrm{AlH}_{3}$ & & -244.169203 & -1441 & -60 & -63 \\
\hline $\mathrm{SiH}_{4}$ & & -291.839779 & -1312 & 154 & 152 \\
\hline $\mathrm{PH}_{3}$ & & -343.104304 & -1297 & 19 & 18 \\
\hline $\mathrm{H}_{2} \mathrm{~S}$ & & -399.356295 & -1732 & -37 & -48 \\
\hline $\mathrm{HCl}$ & & -460.771786 & -1416 & -28 & -30 \\
\hline Ar & & -527.496357 & -345 & 0 & 0 \\
\hline $\mathrm{H}_{2} \mathrm{O} \ldots \mathrm{HOH}$ & $\left(C_{s}\right)$ & -152.784870 & -561 & -7 & -8 \\
\hline$n-\mathrm{C}_{4} \mathrm{H}_{10}$ & $\left(C_{2 h}\right)$ & -158.332434 & 946 & 52 & 52 \\
\hline$i-\mathrm{C}_{4} \mathrm{H}_{10}$ & $\left(\mathrm{C}_{3 v}\right)$ & -158.333094 & 12 & 20 & 17 \\
\hline$n \cdot \mathrm{C}_{5} \mathrm{H}_{12}$ & $\left(C_{2 v}\right)$ & -197.617597 & 325 & 92 & 92 \\
\hline neo- $\mathrm{C}_{5} \mathrm{H}_{12}$ & $\left(T_{d}\right)$ & -197.618355 & -842 & -191 & -182 \\
\hline $\mathrm{C}_{6} \mathrm{H}_{6}$ & $\left(D_{6 h}\right)$ & -232.128657 & 1155 & -43 & -45 \\
\hline$n-C_{8} H_{18}$ & $\left(C_{2 h}\right)$ & -315.473034 & 1497 & 48 & 50 \\
\hline $\mathrm{C}_{6} \mathrm{H}_{5} \mathrm{NO}_{2}$ & $\left(C_{2 v}\right)$ & -436.619606 & 875 & -14 & -11 \\
\hline $\mathrm{C}_{6} \mathrm{H}_{5} \mathrm{CHCH}_{2}$ & $\left(C_{s}\right)$ & -309.488863 & 178 & -85 & -100 \\
\hline$n-\mathrm{Si}_{4} \mathrm{H}_{10}$ & $\left(C_{2 h}\right)$ & -1163.841584 & -7814 & 192 & 207 \\
\hline$i-\mathrm{Si}_{4} \mathrm{H}_{10}$ & $\left(C_{3 v}\right)$ & -1163.842281 & -6854 & 276 & 289 \\
\hline$n-\mathrm{Si}_{5} \mathrm{H}_{12}$ & $\left(C_{2 v}\right)$ & -1454.509624 & -7589 & 259 & 248 \\
\hline neo-Si ${ }_{5} \mathrm{H}_{12}$ & $\left(T_{d}\right)$ & -1454.511858 & -7778 & 420 & 443 \\
\hline $\mathbf{P}_{4}$ & $\left(T_{d}\right)$ & -1365.375677 & -7590 & -58 & -26 \\
\hline $\mathbf{S}_{\mathbf{8}}$ & $\left(D_{4 d}\right)$ & -3185.429051 & -7739 & 128 & -3 \\
\hline
\end{tabular}

7) Computed at the B-LYP/6-31 G* level of theory,

b) In hartree for $(96,32,64)$; in $\mu$ hartree relative to $(96,32,64)$ for others.

c) $\mathrm{MP2} / 6-31 \mathrm{G}^{*}$ geometries for systems with up to one heavy atom; HF/6-31 G* geometries for systems with two or more heavy atoms.

${ }^{d}\left(N^{r}, N^{\Omega}\right)$ is an Euler-Maclaurin-Lebedev grid with $N^{r}$ radial and $N^{\Omega}$ angular points; $\left(N^{r}, N^{\star}, N^{\phi}\right)$ is a Murray-Handy-Laming grid with $N^{\prime}$ radial, $N^{\bullet}$ theta and $N^{\oplus}$ phi points.

range of molecular systems. It follows from this that SG-1 yields acceptable grid errors compared with the benchmark grid and, hence, that SG-1 represents a useful compromise between accuracy and expense. It is evident that SG-1 generally exhibits larger total energy grid errors for molecules containing secondrow atoms ( $\mathrm{Na}-\mathrm{Ar}$ ) but much of this error comes from the core region and should not adversely affect quantities of chemical interest. For example, the SG1 errors in the isomerization energies of butane and pentane are comparable to those of the corresponding silanes. We also note that, although the SG-1 and $(30,86)$ grids contain similar numbers of significant grid points [34], SG-1 yields much smaller grid errors.

We will discuss the efficient implementation of the SG-1 grid within the Q-Chem program elsewhere [34]. It is our intention to use this grid as our standard in the future. 


\section{Acknowledgement}

We are grateful to Professor N.C. Handy for preprints of refs. [21,33]. BGJ thanks the Mellon College of Science for a Graduate Fellowship. This research was partly supported by the National Science Foundation (Grant No, 8918623).

\section{Appendix A. The standard nuclear orientation}

The full problem of nuclear orientation is: given a molecule specified by nuclear positions, choose a unique set of Cartesian axes. We shall not attempt to give a complete definition, but limit ourselves to defining an origin and a set of three perpendicular lines through that origin among which Cartesian axes are chosen. That is, we do not attempt to define which of the three is $X, Y$ or $Z$ in all cases, nor do we always define positive versus negative directions. Since the standard atomic grids used all have octahedral symmetry about the individual atoms, the final energy is invariant to such choices.

The proposed orientation rules are as follows:

(1) The origin is always chosen to lie at the center of nuclear positive charge.

(2) If the three principal moments of positive charge (relative to the origin) are unequal, the three corresponding principal axes are used as Cartesian axes. This deals completely with the general asymmetric top molecule.

(3) For nonlinear molecules with symmetry corresponding to a symmetric (but not spherical) top, there will be a unique principal axis, taken to be $Z$. It remains to specify one further axis $(Y)$. To do this, we introduce the concept of a "circular set", composed of equivalent atoms lying in a plane perpendicular to the principal $(Z)$ axis. In general, there will be several circular sets. Of these, we select the "key circular set" by carrying out the following tests successively until any ambiguity is removed:

(a) Which set is nearest the $X Y$ plane?

(b) Which set has a positive projection on the $Z$ axis?

(c) Which set is nearest to the $Z$ axis?

(d) Which set is comprised of atoms with the lowest atomic number?

The "key atom" is an arbitrary member of the key circular set. The $Y Z$ plane is chosen to include this atom. For molecules with two identical moments of positive charge for accidental, rather than symmetry, reasons, the same rules may be used, it being noted that circular sets may then contain only one atom.

(4) For molecules with tetrahedral, octahedral or icosahedral symmetry (spherical tops), the moments of positive charge cannot be used to fix the Cartesian axes. For tetrahedral systems (point groups $T, T_{d}, T_{h}$ ), we choose the three twofold rotation axes. For octahedral systems (point groups $\mathrm{O}, \mathrm{O}_{\mathrm{h}}$ ), we choose the three fourfold axes. Finally, for the icosahedral groups, we arbitrarily select one of the $C_{5}$ axes as the $Z$ axis and then treat the molecule as a symmetric top.

(5) For linear molecules, the $Z$ axis is chosen to lie along the molecular axis. The $Y$ axis is chosen arbitrarily. For single atoms, no specification is necessary.

\section{Appendix B. The standard electronic orientation}

As noted in section 1, complete specification of a DFT model requires us to locate the individual components of degenerate molecular orbitals in a unique manner. It should be noted that this is also true of the complete specification of a Hartree-Fock (HF) model: although the HF energy is unaffected by molecular orbital orientation, this obviously does not apply to the HF wavefunction. We treat the problem in two stages:

Stage 1. If the molecular point group is non-abelian (so that degeneracies occur), imposition of a grid with local octahedral symmetry at each atom may lower the symmetry to the grid point group (a subgroup of the molecular point group). Table B.1 lists these relations. For many of the molecular point groups, the grid lifts the degeneracies and the KohnSham orbitals are automatically classified according to the irreducible representations of the grid point group. In the remaining cases (which include gridless computations such as HF), it is necessary to proceed to the next stage.

Stage 2. When stage 1 is insufficient, the problematic degeneracies can then be lifted by the imposition of a small, perturbing quadrupole field. We have found that a field with $x^{2}, y^{2}$ and $z^{2}$ components of $+10^{-10},+2 \times 10^{-10}$ and $-3 \times 10^{-10}$ atomic 
Table B.I

Reduction of symmetry by imposition of octahedral grid

\begin{tabular}{|c|c|c|c|}
\hline \multicolumn{2}{|c|}{ Abelian grid point group } & \multicolumn{2}{|c|}{ Non-abelian grid point group } \\
\hline $\begin{array}{l}\text { molecular } \\
\text { point group }\end{array}$ & $\begin{array}{l}\text { grid } \\
\text { point group }\end{array}$ & $\begin{array}{l}\text { molecular } \\
\text { point group }\end{array}$ & $\begin{array}{l}\text { grid } \\
\text { point group }\end{array}$ \\
\hline $\mathrm{C}_{2 n+1}$ & $\mathrm{C}_{1}$ & $\mathrm{C}_{4 \pi}$ & $\mathrm{C}_{4}$ \\
\hline $\mathrm{C}_{2 n+1 . v}$ & $\mathrm{C}_{3}$ & $\mathrm{C}_{4 n v}$ & $\mathrm{C}_{4 v}$ \\
\hline $\mathrm{C}_{2 n+1, \mathrm{~h}}$ & C. & $\mathrm{C}_{\text {Anth }}$ & $\mathrm{C}_{\boldsymbol{\Delta h}}$ \\
\hline $\mathrm{D}_{2 n+1}$ & $\mathrm{C}_{2}$ & $\mathrm{D}_{4 n}$ & $\mathrm{D}_{4}$ \\
\hline $\mathrm{D}_{2 n+1, \mathrm{~h}}$ & $\mathrm{C}_{2 \mathrm{v}}$ & $\mathrm{D}_{4 n \mathrm{~h}}$ & $D_{4 h}$ \\
\hline $\mathrm{D}_{2 n+1, \mathrm{~d}}$ & $\mathrm{C}_{2 \mathrm{~h}}$ & $D_{4 n d}$ & $\mathrm{D}_{4 \mathrm{~d}}$ \\
\hline $\mathrm{C}_{4 n+2}$ & $C_{2}$ & $\mathrm{D}_{4 n+2, \mathrm{~d}}$ & $\mathrm{D}_{2 \mathrm{~d}}$ \\
\hline $\begin{array}{l}\mathrm{C}_{4 n+2} \\
\mathrm{C}_{4 n+2, \mathrm{v}}\end{array}$ & $\begin{array}{l}\mathrm{C}_{2} \\
\mathrm{C}_{2 \mathrm{v}}\end{array}$ & $\mathrm{S}_{4 n}$ & $\mathrm{~S}_{4}$ \\
\hline $\mathrm{C}_{4 n+2, \mathrm{~h}}$ & $\mathrm{C}_{2 \mathrm{~h}}$ & $\mathbf{T}$ & $\mathbf{T}$ \\
\hline $\mathrm{D}_{4 n+2}$ & $\mathrm{D}_{2}$ & $T_{d}$ & $T_{d}$ \\
\hline $\mathrm{D}_{4 n+2, h}$ & $\mathrm{D}_{\mathbf{2 h}}$ & $\mathbf{T}_{\mathbf{h}}$ & $T_{h}$ \\
\hline \multirow[t]{7}{*}{$S_{4 n+2, d}$} & $\mathrm{C}_{\mathrm{i}}$ & 0 & 0 \\
\hline & & $\mathrm{O}_{\mathrm{h}}$ & $\mathrm{O}_{\mathrm{h}}$ \\
\hline & & I & $C_{2}$ \\
\hline & & $I_{\mathrm{h}}$ & $\mathrm{C}_{2 \mathrm{~h}}$ \\
\hline & & $\mathrm{C}_{\infty o v}$ & $\mathrm{C}_{4 v}$ \\
\hline & & $D_{\infty h}$ & $\mathrm{D}_{4 \mathrm{~h}}$ \\
\hline & & $\mathbf{K}_{\mathbf{h}}$ & $O_{h}$ \\
\hline
\end{tabular}

units, respectively, yields satisfactory results in most cases. The effect of such a field on the molecular total energy is negligible and, indeed, is comparable to the errors which are introduced by integral cutoffs.

\section{References}

[1] P. Hohenberg and W. Kohn, Phys. Rev. B 136 (1964) 864; W. Kohn and L.J. Sham, Phys. Rev. A 140 (1965) 1133.

[2] R.G. Parr and W. Yang, Density-functional theory of atoms and molecules (Oxford, New York, 1989).

[3] J.K. Labanowski and J.A. Andzelm, eds., Density functional methods in chemistry (Springer, Berlin, 1991).

[4] T. Ziegler, Chem. Rev. 91 (1991) 651.

[5] P.A.M. Dirac, Proc. Cambridge Phil. Soc. 26 (1930) 376.

[6] S.J. Vosko, L. Wilk and M. Nusair, Can. J. Phys. 58 (1980) 1200.

[7] A.D. Becke, Phys. Rev. A 38 (1988) 3098.

[8] C. Lee, W. Yang and R.G. Parr, Phys. Rev. B 37 (1988) 785 ;

B. Miehlich, A. Savin, H. Stoll and H. Preuss, Chem. Phys. Letters 157 (1989) 200.

[9] L.C. Wilson and M. Leyy, Phys. Rev. B 41 (1990) 12930.

[10] J.P. Perdew, in: Proceedings of the 21 st Annual International Symposium Electronic Structure of Solids (Nova Science, Commack, NY), in press.
[11] A.D. Becke, Numol, Intern. J. Quantum Chem. Symp. 23 (1989) 1280 .

[12] B. Delley, DMol, J. Chem. Phys. 92 (1990) 508.

[13] D.R. Salahub, DeMon, in: Density functional methods in chemistry, eds. J.K. Labanowski and J.W. Andzelm (Springer, Berlin, 1991).

[14] J. Andzelm and W. Wimmer, DGauss, J. Chem. Phys. 96 (1992) 1280.

[15] R.D. Amos, I.L. Alberts, J.S. Andrews, S.M. Colwell, N.C. Handy, D. Jayatilaka, P.J. Knowles, R. Kobayashi, N. Koga, K.E. Laidig, P.E. Maslen, C.W. Murray, J.E. Rice, J. Sanz, E.D. Simandiras, A.J. Stone and M.-D. Su, Cadpac 5, Cambridge (1992).

[16] P.M.W. Gill, B.G. Johnson and C.A. Gonzalez, Q-Chem (QChem, Inc., Pittsburgh, PA, 1993).

[17] A.D. Becke, J. Chem. Phys. 88 (1988) 2547

[18] M.J. Frisch, G.W. Trucks, M. Head-Gordon, P.M.W. Gill, M.W. Wong, J.B. Foresman, B.G. Johnson, H.B. Schlegel, M.A. Robb, E.S. Replogle, R. Gomperts, J.L. Andres, K. Raghavachari, J.S. Binkley, C. Gonzaiez, R.L. Martin, D.J. Fox, D.J. DeFrees, J. Baker, J.J.P. Stewart and J.A. Pople, GAUSSIAN 92 (Gaussian, Inc, Pittsburgh, PA, 1992).

[19] J.A. Pople, in: Energy, structure and reactivity, eds. D.W. Smith and W.B. McRae (Wiley, New York, 1971).

[20] A.H. Stroud, Approximate calculation of multiple integrals (Prentice-Hall, Englewood Cliffs, 1971).

[2I] C.W. Murray, N.C. Handy and G.J. Laming, Mol. Phys. 78 (1993) 997.

[22] V.I. Lebedev, Zh. Vychisl. Mat. Mat. Fiz. 15 (1975) 48; Vychisl. Mat. Mat. Fiz. 16 (1976) 293; Sibirsk. Mat. Zh. 18 (1977) 132; Proc. Conf. Novosibirsk (1978), ed. S.L. Sobolev (Nauka Sibirsk. Otdel., Novosibirsk, 1980).

[23] S.I. Konyaev, Mat. Zametki 25 (1979) 629.

[24] Handbook of Mathematical Functions, eds. M. Abramowitz and I.A. Stegun (Dover, New York, 1970).

[25] W. Yang, J. Chem. Phys. 94 (1991) 1208.

[26] N.C. Handy and S.F. Boys, Theoret. Chim. Acta 31 (1973) 195.

[27] P.M.W. Gill, B.G. Johnson, J.A. Pople and M.J. Frisch, Chem. Phys. Letters 197 (1992) 499.

[28] B.G. Johnson, P.M.W. Gill and J.A. Pople, J. Chem. Phys. 97 (1992) 7846.

[29] B.G. Johnson, P.M.W. Gill and J.A. Pople, J. Chem. Phys. 98 (1993) 5612.

[30] P.M.W. Gill, B.G. Johnson, J.A. Pople and M.J. Frisch, Intern. J. Quantum Chem. Symp. 26 (1992) 319.

[31] J.C. Slater, Phys. Rev. 36 (1930) 57.

[32] J.A. Pople, P.M.W. Gill and B.G. Johnson, Chem. Phys. Letters 199 (1992) 557.

[33] N.C. Handy, P.E. Maslen, R.D. Amos, J.S. Andrews, C.W. Murray and G.J. Laming, Chem. Phys. Letters 197 (1992) 506.

[34] P.M.W. Gill, B.G. Johnson and J.A. Pople, manuscript in preparation. 